\title{
Dosimetry of cone beam computed tomography $(C B C T)$; the current status and future prospects
}

\author{
Volume 7 Issue I - 2017 \\ Mohammed Al Towairqi \\ Taif University, Saudi Arabia
}

\section{Literature Review}

Cancer is a major cause of death worldwide claiming 8.2million lives as reported by World Health Organization (WHO) in 2012. Cancer treatment has been modernised with sophisticated technology to provide patients with the highest level of treatment. Radiotherapy has recently become one of the most common techniques used to treat people with cancer. It uses high energy beams- normally $\mathrm{x}$-rays, photons, or electrons- to kill the tumour by damaging the cell's DNA. ${ }^{2}$ Recently, radiotherapy has been immensely developed by being integrated with modern imaging modalities. One of these modalities is Computed Tomography (CT) which has been used in the clinical practice for diagnostic purposes. With the recently developed techniques in cancer treatment, CT has effectively been integrated with radiotherapy to facilitate the procedures of cancer treatment by visualizing the tumour size and location as so called Image Guided Radiation Therapy (IGRT). Cone Beam Computed Tomography $(\mathrm{CBCT})$ for example is an imaging technique that provides three dimensional (3D) images of tumour anatomy and target volume treated by IGRT. This chapter will first introduce Cone Beam CT and its current applications in the medical field. Then, it will describe Cone Beam CT dosimetry in IGRT. After that, it will discuss some of the relevant works which have been done by other researchers. Finally, it will suggest some future work needed and the research gaps recommended for further investigation.

\section{Cone beam computed tomography (CBCT)}

CBCT is defined by Raut Dessai et al., ${ }^{3}$ as ${ }^{~} \mathrm{CBCT}$ is a digital imaging modality that presents computed three-dimensional data in two-dimensional slices from any plane and at low radiation exposure compared to conventional CT'. Another definition by Miracle \& Mukherji ${ }^{4}$ is that 'Cone Beam CT is an advancement in CT imaging that has begun to emerge as a potentially low-dose cross-sectional techniques for visualizing bony structures in the head and neck'. Cone Beam CT is considered as a new generation of CT which has been increasingly used in some clinical scenarios such as dentistry and radiography applications. ${ }^{3,5} \mathrm{Khan}^{6}$ highlighted that $\mathrm{CBCT}$ could be formed into two main types as Kilovoltage Cone-Beam CT imager (KVCBCT) and Megavoltage Cone Beam CT (MVCBCT) imager. These two types are now commercially and clinically available within radiology departments according to the objectives of clinical use. By comparison, better soft tissue contrast is obtained by kilovoltage imagers whereas poor contrast can be noticed in megavoltage imagers. In contrast, Megavoltage images are free from streaking artifacts caused by metallic objects, and hence higher quality images are acquired. Khan ${ }^{6}$ also mentioned each CBCT system has its unique advantages and therefore both systems are complementary. Figure 1, shown below, illustrates the distinctive geometry of CBCT compared to conventional fan-beam $\mathrm{CT}$.

\section{History of CBCT}

By extensively investigating any phenomenon and looking for
Correspondence: Mohammed AI Towairqi, Taif University, Airport Road Al Hawiyah Taif, Saudi Arabia, Tel +966597707063, Emailm.altowairqi@tu.edu.sa

Received: January 29, 2016 | Published: January 09, 2017

learning the secrets behind, it is preferable to give a brief history of its technology and gradual developments. CBCT technology has been originated due to successive improvements on imaging modalities over time. Starting with the discovery of Roentgens' rays (x-rays) in $1895^{7}$ and then efforts were made on improving the imaging modalities. Such great efforts and improvements eventually led to the introduction of the first CT scanner which was invented by the British Engineer Sir Godfrey Hounsfield in 1972. ${ }^{3,8}$ High cost and radiation exposure of CT have allowed for more efforts to look for an imaging technique that is capable of providing the identical advantage of CT. However, low cost, less radiation exposure, and short scanning time were the main interests of scientists whose contributions have led to the introduction of Cone Beam CT. ${ }^{9}$ According to Govila, first Cone Beam CT (CBCT) scanner was constructed by Robles $\mathrm{RA}^{10}$ and in 1982 was adapted for clinical use at the Mayo Clinic Biodynamic Research Laboratory. ${ }^{4}$ Sykes et al., ${ }^{11}$ stated that CBCT systems have been available in radiotherapy treatment room after the publication of Jaffray et al. who firstly introduced its concept in 1998 and then becoming commercially available in 2005. As mentioned by Miracle and Mukherji that the first use of $\mathrm{CBCT}$ was in radiation therapy guiding in 1992 and the first CBCT system was fully integrated with the linear accelerator in 1999. ${ }^{4}$ Moreover, early versions of CBCT scanner for dental practice were described by Mozzo et al. and Arai in the late 1990s. ${ }^{4}$ They also illustrated that CBCT has had the potential to be effectively used in imaging of dentistry clinical applications including dentomaxillofacial imaging, implantology, orthodontics, and endodontics. ${ }^{4}$ Hyer has stated that $\mathrm{CBCT}$ has gained remarkable popularity and become highly demanded among the clinical set. As a result, Elekta and Varian have recently offered medical linear accelerators, used in the delivery of therapeutic doses, integrated with kilovoltage CBCT systems. These two currently available types of gantry mounted kilovoltage CBCT namely On Board Imager 'OBI, Varian Medical Systems', and X-ray Volumetric Imager 'XVI, Elekta Oncology Systems'. ${ }^{12,13}$ 


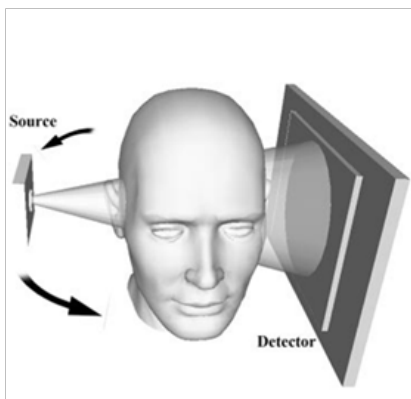

A

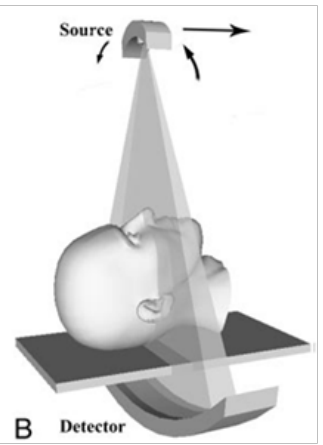

Figure I Illustration of the geometrical variations between Cone Beam CT (CBCT) and fan-beam CT. Left-hand side represents Con Beam CT; right-hand side represents fan-beam $\mathrm{CT}^{5}$

\section{Radiation protection principles}

Ionizing radiation has a negative impact of on the human body. There was an urgently need to create an independent, international, and non-governmental organization that is highly concerned with radiation protection named International Commission on Radiation Protection (ICRP). It aims to provide recommendations and guidance on radiation protection for the environment \& humans involved in radiation activities. It also aims to prevent common side effects associated with radiation exposure such as cancer. The main task of ICRP has led to introduce radiation protection principles that are highly recommended before conducting any radiation activity in both medical and industrial practices. ${ }^{14}$ The radiation protection principles have been reported by ICRP in their annual reports and Australian Radiation Protection and Nuclear Safety Agency (ARPANSA). ARPANSA is an Australian governmental body that provides the system of radiation protection in Australia which is directly derived from the recommendation of ICRP. According to ARPANSA, radiation protection principles must be applied prior to performing any radiation practice. The general principles are justification, optimization, and dose limitation. By being investigated in more depth, justification means that the benefits of conducting a radiation activity are outweigh the risks might be caused by being exposed to radiation. In addition, optimization ensures that individual doses and number of people exposed to radiation follow the radiation principle As Low As Reasonably Achievable (ALARA). Lastly, limitation means radiation doses to individuals involved in the justified activities must fall below acceptable dose limits and doses always are kept limited. ${ }^{15}$

\section{Radiation doses caused by imaging modalities}

Imaging doses have been considered to be a clinical concern and must be minimised and controlled by professionals and care providers among the medical practice. Radiation protection principles must be involved and applied in medical scenario to protect both patients and staff. The ICRP recommends that excess doses of imaging modalities must be justified, optimized, and minimised to the lowest range according to the radiation safety protocol ALARA. ${ }^{16}$ As stated by $\mathrm{Khan}^{6}$ that 'excessive dose from intensive imaging procedures is a serious problem in IGRT",

Clinical and empirical investigations have shown that CBCT has a negative impact on patients, undergoing IGRT, as they receive additional doses more than the prescribed dose for treatment purposes during radiotherapy treatment sessions. Sykes et al., ${ }^{11}$ have discussed in his literature that CBCT, used in IGRT, was initially introduced in some countries regardless the additional doses delivered to the patients. It was justified that the benefits of IGRT outweigh the risk of the $\mathrm{CBCT}$ dose. Though, worrying statements have been made for the amount of CBCT doses by some publications in regard to this matter. Spezi et al. have investigated around forty fractions of imaging the head and neck for radiotherapy purposes. They found that a routine daily use of CBCT during radiotherapy, supposing a sum of forty scans, might result in additional mean dose ranged from 1-2 Gy with utmost doses ranged from 3-7 Gy.

In addition, Rehan, ${ }^{17}$ a member of an ICRP committee, asserted in his talk during annual meeting of ICRP members in 2013 that 'CT is going to be a major source of radiation exposure to population'. He also confirmed that using $\mathrm{CBCT}$, for radiotherapy guiding, results in overall dose to a patient around $40 \mathrm{mGy}$ per fraction due to CBCT imaging. As a consequence, this would result in a total imaging dose of $1.4 \mathrm{~Gy}$, over 35 fractions for the whole treatment sessions, which is not ignorable. Hyer and Hintenlang ${ }^{12}$ have discussed that despite the explicit benefits of $\mathrm{CBCT}$ as an imaging technique in improving the patient positioning and dose verification, it still has a negative side effect by depositing additional radiation dose which could lead to a total dose of a Gray or more to body organs especially if used on a daily basis.

More attention must be paid to paediatric patients who receive additional imaging doses as they are more sensitive to radiation than adults. As reported by Sykes et al., ${ }^{11}$ increased interest has been shown by a recent campaign which aims to provide radiation protection at paediatric practice and applying the principles of As Low as Reasonably Practicable (ALARP). According to the recent literature published by Sykes, a research study conducted by Ding et al. dose calculation was performed in comparison between paediatric patients and adults. It has been observed that paediatric patients receive doses two times higher than that of adults.

\section{Measuring of computed tomography (CT) dose}

Understanding the concept of $\mathrm{CBCT}$ dose measurement requires a comprehensive understanding of dose measurement in conventional fan-beam Computed Tomography (FBCT). ${ }^{11}$ The concept of Computed Tomography Dose Index (CTDI) was initially suggested by Jucius and Kambic and then established by Shope et al. CTDI ${ }_{100}$ has been widely known and accepted as the standard CT dose descriptor which uses the $100 \mathrm{~mm}$ long cylinder-shaped ionisation chamber in a cylinder-shaped phantom. $C T D I_{100}$ can be expressed in Equation (1) as mentioned below. ${ }^{11,13}$

$$
C T D I_{100}=\frac{1}{n \cdot T} \cdot \int_{-50 M M}^{+50 M M} D(z) d z
$$

where, $n$ is the quantity of slices; $T$ is the slice thickness; $D(z)$ is the dose profile along the axial plane.

However, average dose in the axial plane could be estimated as so called weighted CTDI. In addition, equation (2) is derived from measurements averaged at the phantom's centre and the periphery used known as $C T D I_{w}$. Moreover, it calculates the average dose in a plane that is exposed to a number of of contiguous axial scans that equal to pitch of unity. $C T D I_{w}$ can be expressed as follows: ${ }^{11}$

$$
C T D I_{w}=1 / 3 C T D I_{\text {centre }}+2 / 3 C T D I_{\text {periphery }}
$$

In contrast, the case becomes different for a helical scan with nonunity pitch. Hence, volume $C T D I$ has been introduced and accepted for 
such measurement. It can be calculated by dividing weighted CTDI on pitch factor. $C T D I_{v o l}$ can be expressed as follows: ${ }^{11}$

$$
C T D I_{v o l}=\frac{C T D I_{w}}{\operatorname{pitch}(m G y)}
$$

where, "pitch is the table increment per revolution as a fraction of the detector width (nT)".

The following expression represents the concept of Dose Length Product (DLP). It can be acquired when multiplying $C T D I_{v o l}$ by the scan length, as follows:

$$
D L P=C T D I_{v o l} \times L
$$

Where, $\mathrm{L}$ is the length of the scan

Unlike conventional fan beam CT, distinguished geometry of $\mathrm{CBCT}$ has made some changes in terms of CBCT dose measurements. In case of CBCT dose, CTDI concept is no longer a reliable measurement technique as cone angle increases unlike fan beam CT angle. The first reason of this is due to the fact that the non-uniformity of the dose profile along the central axis. The second reason is that the dose profile tails exceed the $10 \mathrm{~cm}$ length of the ion chamber used. The third reason is that the phantom becomes inadequate to detect the whole beam width. The fourth reason is that the increment of central and peripheral dose in $C T D I_{w}$ does not show an accurate prediction of the average dose across the volume. ${ }^{11}$ The next section will describe the concept of CBCT dose index (CBCTDI) in more details.

\section{Measuring of cone beam computed tomography (CBCT) dose}

The concept of CBCT dose index $\left(\mathrm{CBCTDI}_{w}\right)$ was introduced following the introduction of $\mathrm{CBCT}$ and knowing that CTDI is not an accurate estimate in $\mathrm{CBCT}$. In regard to $\mathrm{CBCT}$ imaging technique, beam geometries have been adapted in the form of cone in order to acquire 3D images of the organs with maximum dose at the centre and minimum dose at the periphery. With notable geometrical differences between conventional fan beam CT and CBCT, it is been assumed that CTDI concept is no longer becomes a reliable technique to accurately measure the dose in CBCT due to the geometrical changes adopted such as the chamber and phantom's specific lengths. ${ }^{11,19}$ Moreover, Cheng et al., ${ }^{18}$ has highlighted that many authors have recently suggested the adoption of the $C T D I_{w}$ concept, used in conventional $\mathrm{CT}$, to be an appropriate method in estimate the CBCT dose. This is as so called weighted cone beam $\mathrm{CT}$ dose index $\left(\mathrm{CBCTDI}_{w}\right)$, and can be defined as follows:

$$
C B C T D I w=(1 / 3) D_{0}+(2 / 3) D_{P}^{-}
$$

where, $\mathrm{D}_{0}$ is the central axis dose,

$D_{P}^{-}$is the mean peripheral dose in the phantom at different angles.

\section{Conducting actual CBCT dose measurements}

Cone Beam CT dosimetry is a widespread area and now being a hot topic of scientific papers that have been recently published. Research studies have deeply investigated the CBCT dose of patients who have undergone IGRT. Some dose measurements have been done by performing actual measurements on commercially available phantoms (i.e. anthropomorphic phantoms - homemade phantoms). According to Kim et al., ${ }^{19}$ imaging doses, using kilovoltage CBCT during radiotherapy, were investigated using selected dosimeters and body phantoms at three different treatment sites; the head and neck, chest, and pelvis. The authors have measured primary radiation doses using a glass dosimeter inserted at different locations of a homemade phantom with standard imaging options. Dose measurements have shown that the head and neck, chest, and pelvis have absorbed doses of 1.9, 5.1, and 16.7 cGy respectively. However, secondary doses were measured per scan during treatment using a glass dosimeter placed at a distance of $20-60 \mathrm{~cm}$ from the isocenter suing an anthropomorphic phantom. Moreover, the secondary dose measurements per scan were averaged to be from 0.67-0.02 cGy for the pelvis, and 0.07 $0.003 \mathrm{cGy}$ for the head and neck. It is apparent that there is a bigger secondary dose associated with CBCT for treatment of the pelvis region than that dose of $\mathrm{CBCT}$ for treatment of the head and neck region. Another research study, conducted by Kan et al. ${ }^{20}$ has shown that comprehensive dose measurements were performed including absorbed doses and effective doses from CBCT for some different body sites; head and neck, chest, and pelvis. The authors performed these measurements using Thermoluminescent dosimeter (TLD) in a female anthropomorphic phantom using CBCT system On-Board Imager (OBI). Furthermore, effective doses were reported to the body, and 26 organs were measured for their absorbed dose using two CBCT scanning mode settings; low-dose mode and standard mode. They also performed comparisons of patient doses and image quality with the two CBCT acquisition modes mentioned previously. Results show that the head and neck, chest, and pelvis receive absorbed dose of $6.7,4.6$, and $5.4 \mathrm{cGy}$ respectively which results from the skin dose using standard CBCT dose Also, the head and neck, chest, and pelvis receive effective dose of $10.3,23.7,22.7 \mathrm{mSv}$ respectively which is a consequence from the body dose using standard mode. The significant results are that patient's doses from low-dose mode are much lower than standard mode with approximately one fifth.

\section{Performing simulated CBCT dose measurements}

However, dose measurements have also been performed by simulating the imaging process depending on highly sophisticated programming languages. Monte Carlo (MC) is a well-known programming language that has now been used in clinical scenario for such purposes. According Kim et al., ${ }^{13}$ dosimetry in Kilovolatege $\mathrm{CBCT}$ is a challenge as the physical measurements are limited. Therefore, CT dose index (CTDI) and dose length product (DLP) were estimated depending on $\mathrm{MC}$ by using a Varian On-Board Imager (OBI). In addition, two CT phantoms, head and body, were created using BEAMnrc/EGSnrc. Another MC user code (DOSXYZnrc) was used to simulate around twenty CBCT scans for the some beam widths. Their results showed that DLP was proportional to the beam collimation, CTDIw, 600 has a value of $8.74 \mathrm{cGy}$ for head scan, and CTDIw, 900 has a value of 4.26 cGy for body scan. Another significant result is that differences of CTDIw values were successfully evaluated within 3\% difference between MC simulations and point dose measurements.

\section{Risk of radiation-induced cancer (secondary cancer)}

Risk of getting a secondary cancer, or so called radiation-induced cancer, has been investigated by many researchers in their latest published papers. Kan et al., ${ }^{20}$ discussed the assessment of radiationinduced cancer due to organ dose from Kilovoltage CBCT. Lifetime attributable risk (LAR), excess absolute risk (EAR), and excess relative risk (ERR) were all estimated relying on Biological Effect of Ionizing Radiation (BEIR) VII models. It is clearly shown that using $\mathrm{CBCT}$ in Image Guided Radiation Therapy (IGRT) increases the 
lifetime cancer risk while positioning patients during treatment. It was reported that around 400 per 10,000 persons are at cancer risk if 30 $\mathrm{CBCT}$ scans are performed for positioning of patients during treatment of pelvis site. ${ }^{19}$ A research study has highlighted that secondary cancer risk could be increased by up to $2 \%$ to $4 \%$ if using standard mode CBCT for patient's position verification on a daily basis. However, using the low-mode dose can decrease the effective dose to the body and the risk of the secondary cancer. As reported, adjustments of some CBCT scan settings are recommended. Using law-dose mode or lower $\mathrm{mAs}$ could be considered for daily CBCT where soft tissue is the not the main interest especially when visualizing certain treatment sites such as bony anatomy.

\section{Research gap \& possible future work}

Literature has shown some possible future work and efforts that should be directed to achieve some work as possible. Firstly, dose reduction strategies are required to be investigated with keeping the image quality high enough to ensure the delivery of the accurate dose during treatment course per fraction. A research study conducted by Kim et al., ${ }^{19}$ has recommended that future work should be directed to improve the image quality with keeping radiation doses low. Moreover, Sykes et al., ${ }^{11}$ recommend in his recent literature review paper published that:

There is a great deal of work required to both improve CBCT technology and its image quality capabilities to minimise the radiation dose required and understand the level of image quality required for image guidance which in some cases can be performed accurately by automatic image registration algorithms with much less dose than would be desirable for a human operator to perform manual image registration.

Secondly, risk of radiation-induced cancer (secondary cancer) should be accurately estimated, evaluated, and assessed. Kim et al., ${ }^{19}$ recommend that accurate estimation of radiation-induced cancer risk should be obtained and more investigated. Sykes et al., ${ }^{11}$ report that there is still uncertainty in expecting the risk of the secondary malignancy due to the fact that the insufficient epidemiological data and poor understanding of the mechanisms for cancer development. Khan ${ }^{6}$ recommends in his newly published book that:

There is a need to evaluate stochastic as well as nonstochastic risks involved in all the imaging procedures used in radiation therapy. Imaging doses must be balanced with demonstrable improvements in the delivery of therapeutic dose.

Thirdly, accurate organ doses should be calculated and more correlated with the risk to have accurate risk estimation. Sykes et al., ${ }^{11}$ recommend that once the additional risks of the imaging doses are determined, then there is a need to calculate accurate patient organ doses. The main reason so that it can be easily correlated with the risk that would lead to better risk estimation.

Fourthly, CBCT has been a hot topic worldwide and was given a special attention during the annual ICRP meeting held in Dubai 2013. Rehani, ${ }^{17}$ a member of an ICRP committee, asserted that tissue reactions from Cone Beam CT (CBCT) have not been described among both workers and patients. He also emphasized that some steps are expected from the industry. For example, alerts \& notifications should be provided when dose gets higher than chosen. Also, exposure should be stopped at certain levels if normal is exceeded. On the other hand, valuable recommendations were given for industry and manufacturers of CBCT systems to improve the outcomes. Some of the recommendations are as follows:
1. Radiation output throughout the examination should be regularly monitored,

2. Radiation output should be comparing with reference or desired levels,

3. Feedback could be provided to system so that it can be automatically adjusted.

4. Risk could be properly managed and assessed.

However, CBCT is identical to any new technology that has unique advantages but still has some limitations that need to go for further improvements. Rehani ${ }^{17}$ has also pointed out some important actions that need to be taken to improve the outcomes CBCT systems in the clinical practice. One important point is that current CBCT scanners do not provide values in standardised dose index for patient or machine. It has been suggested that some actions need to be taken in the future. There is a necessity to develop approaches that aggregate exposures to individual patients during whole procedures as currently $\mathrm{CBCT}$ equipment presents a new challenge in dosimetry. Another important suggestion is that training of personnel, operating $\mathrm{CBCT}$ for diagnostic purpose, is more recommended in the identical way as for diagnostic CT. As recommended by ICRP that 'the level of training in radiological protection should be commensurate with the level of expected radiation exposure'.

Lastly, by investigating the literature in more depth, it is clearly shown that there is no abundant $\mathrm{CBCT}$ research within the Middle Eastern clinical community especially in Saudi Arabia. By covering the scope of the undergoing research study, it is obvious that there has been a lack of CBCT research in terms of doses, an accurate estimation of the risk of secondary cancer caused by extra doses, and the like within the clinical practice among Saudi hospitals. This surely results in not providing qualified staff in dealing with $\mathrm{CBCT}$ systems and fully aware of the amount of secondary doses given to patients during radiotherapy treatment sessions. Hence, cancer patients who undergo IGRT are almost likely to get a higher radiation dose more than prescribed in the treatment plan. Consequently, risk probability of getting radiation-induced malignancy will be definitely increased within local \& clinical community. Therefore, more research on $\mathrm{CBCT}$ and issues related are urgently needed. Moreover, the risk of secondary cancer needs to be accurately assessed and estimated by investigating real patients' data within two diverse clinical \& cultural communities retrospectively. Both Australians and Saudi patients have been chosen as research samples to carry out this research study.

\section{Conclusion}

This paper aims to review the dosimetry of the two CBCT systems available in the clinical scenario. It has also shown introductory information about CBCT, its history, current medical applications, and latest knowledge on CBCT dosimetry. Finally, it has summarized and pointed out some work done previously by other researchers around the world until present. CBCT has now been the preferable choice of professionals in dentistry, radiography, and radiotherapy guiding due to its unique advantages over conventional CT. It also has attracted the interests of many research papers recently published. With all strength points mentioned previously, $\mathrm{CBCT}$ is still undergoing some improvements recommended and promising to be one of the imaging modalities that are globally preferred and effectively used. Future work of CBCT should be directed toward improving the CBCT systems by providing valuable recommendations and technical suggestions to the manufacturers to make some desired improvements to the current system. Also, training of personnel, responsible for CBCT 
systems at radiology departments, is essentially required to improve the mechanism of diagnosis in the way that is commensurate with the international regulations of radiation protection in the medical practice in order to get the high quality of the healthcare which given to patients.

\section{Acknowledgments}

None.

\section{Conflicts of interest}

Author declares there are no conflicts of interest.

\section{References}

1. WHO. Cancer; 2014.

2. Yang G, Nowsheen S, Aziz K, Toxicity and adverse effects of Tamoxifen and other anti-estrogen drugs. Pharmacol Ther. 2013;139(3):392-404.

3. Raut Dessai S, Barretto E, Swamy D. Cone Beam Computed Tomography: A Comprehensive Review. Research \& Reviews: Journal of Medical and Health Sciences. 2014;3:1-10.

4. Miracle AC, Mukherji SK. Conebeam CT of the head and neck, part 2: clinical applications. AJNR Am J Neuroradiol. 2009b;30(7):1285-1292.

5. ADACOS. The use of cone-beam computed tomography in dentistry: an advisory statement from the American Dental Association Council on Scientific Affairs. Journal of the American Dental Association. 2012;143(8):899-902.

6. Khan FM. The Physics of Radiation Therapy; 2014.

7. Lederman M. The early history of radiotherapy: $1895-1939$. Int J Radiat Oncol Biol Phys. 1981;7:639-648.

8. Kalender WA. X-ray computed tomography. Phys Med Biol. 2006;51(13): R29-R43.
9. Miracle AC, Mukherji SK. Conebeam CT of the head and neck, part 1: physical principles. AJNR Am J Neuroradiol. 2009;30(6):1088-1095.

10. Govila S, Gundappa M. Cone beam computed tomography-an overview. Journal of Conservative Dentistry. 2007;10(2):53.

11. Sykes R, Lindsay R, Iball G, et al. Dosimetry of CBCT: methods, doses and clinical consequences. Journal of Physics: Conference Series. 2013;444:012017.

12. Hyer DE, Hintenlang DE. Estimation of organ doses from kilovoltage cone-beam CT imaging used during radiotherapy patient position verification. Med phy. 2010;37(9):4620-4626.

13. Kim S, Song H, Samei E, et al. Computed tomography dose index and dose length product for cone-beam CT: Monte Carlo simulations. J Appl Clin Med Phys. 2011; 12(2): 3395.

14. ICRP. About ICRP; 2014.

15. ARPANSA. Radiation Protection in the Medical Applications of Ionizing Radiation. Australian Government; 2007.

16. Vano E. ICRP recommendations on 'Managing patient dose in digital radiology. Radiat protect dosimetry. 2005;114(1-3):126-130.

17. Rehani M. Patient dose management in $C T$ and $C B C T$. Abu Dhabi; 2013.

18. Cheng HC, Wu VW, Liu ES, et al. Evaluation of radiation dose and image quality for the Varian cone beam computed tomography system. Int J Radiat Oncol Biol Phys. 2011;80(1):291-300.

19. Kim DW, Chung WK, Yoon M. Imaging Doses and Secondary Cancer Risk From Kilovoltage Cone-beam CT in Radiation Therapy. Health physics. 2013;104(5):499-503.

20. Kan MW, Leung LH, Wong W, et al. Radiation dose from cone beam computed tomography for image-guided radiation therapy. Int J Radiat Oncol Biol Phys. 2008; 70(1):272-279. 